\title{
On the mechanical properties of graphyne, graphdiyne and other poly(phenylacetylene) networks
}

Edera P. Degabriele ${ }^{1}$, James N. Grima-Cornish ${ }^{2}$, Daphne Attard ${ }^{1}$, Roberto Caruana-Gauci ${ }^{1}$, Ruben Gatt ${ }^{1}$, Kenneth E. Evans ${ }^{3}$ and Joseph N. Grima ${ }^{*}, 1,2$

${ }^{1}$ Metamaterials Unit, Faculty of Science, University of Malta, Msida MSD 2080, Malta

${ }^{2}$ Department of Chemistry, Faculty of Science, University of Malta, Msida MSD 2080, Malta

${ }^{3}$ College of Engineering Mathematics and Physical Science, Harrison Building, University of Exeter, Exeter EX4 4QF, United Kingdom.

*Corresponding author: email joseph.grima@um.edu.mt, Phone: +356 23402274

Keywords: auxetic; graphene; graphyne; mechanical properties

\begin{abstract}
We simulate, analyse and compare the mechanical properties of a number of molecular sheetlike systems based on fully-substituted, penta-substituted, tetra-substituted and tri-substituted poly(phenylacetylene) using static force-field based methods. The networks are modelled in a 3D environment with and without inter-layer interactions in analogy to graphite and graphene respectively. It is shown that by varying the type of substitution and the length of the acetylene chain, one may control the mechanical properties of such systems. In particular, it is shown that poly(phenylacetylene) systems can be specifically designed to exhibit negative Poisson's ratio, and that the stiffness can be controlled in an independent manner from the Poisson's ratios. This is significant as it highlights the fact that such systems can be tailored to exhibit a particular set of mechanical properties.
\end{abstract}




\section{Introduction}

Auxetics (materials with a negative Poisson's ratio) exhibit the anomalous property of expanding laterally when uniaxially stretched and contracted when uniaxially compressed. Although this property is not commonly encountered in most everyday materials, in the last decades, particularly since the coining of the term 'auxetic' in 1991[1], various previously unknown materials and models which exhibit this property have been discovered and/or developed. These include molecular systems such as zeolites and silicates[2]-[6], liquid crystalline polymers[7], [8], carbon based systems[9]-[12] and cubic metals[13], [14]; microstructured materials such as foams[15]-[20] and microstructured polymers[21]-[24] as well as macromodels based on re-entrant honeycombs[25]-[27], rotating rigid units[5], [28][32], dilational systems[33], [34], non-convex hard bodies[35], [36], chiral honeycombs[5], [37]-[40] and helices[41].

Materials which have been extensively studied vis-à-vis their potential to exhibit a negative Poisson's ratios are those made from phenyl rings connected together via acetylene chains. In particular, Evans et al. [1] had proposed a crystalline network having 1,2,3 tri-substituted phenyl rings connected together via acetylene chains of various lengths, collectively referred to as reflexyne networks as well as their 1,3,5 tri-substituted equivalents, collectively referred to as flexyne networks. The reflexyne networks with their arrow-shaped features were meant to mimic the behaviour of auxetic re-entrant honeycombs[25] whilst the flexyne networks with their Y-shaped joints were meant to mimic conventional honeycombs. These nano-scale networks were modelled as crystalline systems using force field based simulations[1], [42], [43] where it was shown that the sign of the Poisson's ratio is dependent on the way the acetylene chains are connected to the phenyl rings, with the reflexynes exhibiting negative Poisson's ratios for loading along the major directions whilst the flexynes exhibited positive Poisson's ratio. More recently Grima and Evans have also modelled crystalline forms of $1,2,3,4$ tetra-substituted phenyl rings connected together via acetylene chains of various 
lengths, collectively referred to as polytriangles, which were meant to mimic the behaviour of auxetic rotating triangles as well as their fully-substituted equivalents[9]. Here it was shown that the polytriangles have the potential to exhibit negative Poisson's ratios whilst their fully substituted equivalents (i.e. crystalline forms of graphyne, graphdiyne, etc.) exhibit conventional behaviour. Graphyne can be split into three different types[44]; $\alpha$-graphyne, which consists of propyne chains being connected to form a structure of hexagons; $\beta$ graphyne, which uses the same type of chain but forms a structure of hexagons being surrounded by triangles; and $\gamma$-graphyne, which can be described as a structure formed from multiple poly(phenylacetylene) chains, which therefore are composed of carbon atoms in the $\mathrm{sp}$ and $\mathrm{sp}^{2}$ hybridised state[45]. The existence of $\gamma$-Graphyne is permitted due to the ability of carbon to form chains and crystal lattices. It is because of this property that variations of graphyne, such as graphdiyne, which has a similar structure to graphyne but has two triple bonds instead of one between phenyl rings[46], are possible. These systems have primarily been studied as monolayer systems [47]-[52], although, at least in theory it is equally possible to have these systems existing as crystals rather than mono-layers, these being an analogy to graphite as the crystalline equivalent to graphene.

In this respect, it is somewhat unfortunate that the predicted mechanical properties of the crystalline equivalent of the fully-substituted poly(phenylacetylene) systems (i.e. crystalline forms of graphyne, graphidyne, etc.) were first reported merely as a comparison to their auxetic tetra-substituted counterparts, with the result that not much analysis was performed on these systems. Similarly, no attempt was made to analyse the properties of other networks that can be produced through different modes of substitutions. In view of this, an attempt is made to revisit the properties of graphyne, graphdiyne and related sheet-like systems in an attempt to assess the properties that can be obtained from fully-substituted, penta-substituted, tetra-substituted and tri-substituted poly(phenylacetylene) systems shown in Figure 1 in an attempt to identify interesting and anomalous properties that these systems may exhibit. Here 
it must be noted that depending on the manner of substitution, the systems in Figure 1 result in some very different geometries, where for example, the 1,3,5 tri-substituted systems and the $1,2,3,4,5$ penta-substituted networks resemble honeycomb-like structures [53]; the $1,2,4,5$ tetra-substituted networks resemble wine-rack models [54] whilst the 1,2,3,4- tetrasubstituted networks resemble the polytriangles [9]. To simplify discussion a nomenclature system which is based on the manner of substitution of the phenyl rings and the length of the acetylene chains is being proposed, as described in Figure 1. The molecular networks are studied both as single graphene-like layers as well as a system of a collection of stacked graphite-like layers.

\section{INSERT FIGURE 1}

\section{Simulations}

Simulations on the two versions of the systems shown in Figure 1, with $n=1,2,3,4,5$ were carried out using static force-field based simulations within the Cerius ${ }^{2}$ molecular modelling environment using a methodology based on previous studies relating to similar systems $[1,2$ 9]. In all cases a 3D unit cell was used to represent these molecular networks. In the first set, the networks were constructed as graphite-like molecular crystals where the sheets were aligned parallel to the (100) plane of the crystal and allowed to stack in the [100] direction with no additional constraints. In the second set, additional constraints were imposed on the system to model them as graphene-like systems by setting the lattice cell parameters $\beta, \gamma=90^{\circ}$ and $a=200 \AA$. In this setup, the atoms of one layer are superimposable on the corresponding atom of the successive layer and $200 \AA$ apart. This means that the different layers are expected to behave as monolayers since an inter-layer separation of $200 \AA$ de facto means that the different layers are too far apart to interact. 
In all cases, the crystals and monolayers were represented using one of the smaller possible unit cells with a continuum being simulated through the use of periodic boundary conditions. The orientations and unit cells used were as shown in Figure 2 and in all cases, the crystal cells were oriented with respect to the global Cartesian coordinate system, in a manner which forces the [001] crystal direction to remain parallel to the global $z$-axis and the crystal [010] direction aligned in the global $y z$-plane. For the systems where $\beta, \gamma$ were left unconstrained, this alignment leaves the [100] direction free to orient in any direction, whilst for systems where $\beta, \gamma=90^{\circ}$ the [100] direction orients itself with the global $x$-axis.

For all systems, an energy expression was set up, using the PCFF force-field [55]-[57]. The default settings were used with the exception of the non-bond terms which were summed using the Ewald summation method [58]. The atomic positions and appropriate crystal lattice parameters ( $a, b, c, \alpha, \beta, \gamma$ in the case of the crystals; $b, c, \alpha$ in the case of the monolayers) were then optimised so as to minimise the energy of the system to the default Cerius $^{2}$ high convergence criteria which include an RMS gradient less than $0.001 \mathrm{kcal} \mathrm{mol}^{-1} \AA^{-1}$. Following this optimisation procedure, the full $6 \times 6$ stiffness matrix $\mathbf{C}$ and its inverse, the compliance matrix, $\mathbf{S}=\mathbf{C}^{-1}$ were calculated from the second derivative of the potential energy function since:

$$
c_{i j}=\frac{1}{V} \frac{\partial^{2} E}{\partial \varepsilon_{i} \partial \varepsilon_{j}} \quad i, j=1,2, \ldots, 6
$$

where $E$ is the energy expression, $V$ is the volume of the unit cell and $\varepsilon_{i}$ are strain components. This data was then used to calculate the off-axis mechanical properties using standard axis transformation techniques [59]. From the transformed compliances, the off-axis Poisson's ratios $\left(v_{y z}=-s_{32} / s_{22}\right)$, the Young's modulus $\left(E_{y}=1 / s_{22}\right)$ and shear modulus $\left(G_{y z}=1 / s_{44}\right)$ in the $y z$-plane were plotted against $\zeta$, the transformation angle. Finally in an attempt to ensure that the results of the simulations were not an artefact of the size of the unit or the force-field used, the simulations were repeated using Dreiding force-field [60] as implemented in 
Cerius $^{2}$ using the charge equilibration procedure by Rappé et al. [61]. A selection of systems were also re-simulated with the PCFF force-field using larger unit cells $(m \times m \times m$ supercells in the case of crystals, $1 \times m \times m$ supercells in the case of monolayers, $m=2,3,4$.).

\section{Results and discussion}

Images of typical systems obtained after energy minimisation with the PCFF force-field and the simulated mechanical properties in the planes of the networks are shown in Figure 2 (PA1245- $n$ systems in Figure 3 in more detail). Note that all systems minimised to the required convergence criteria and the resulting systems had geometries which, and generally to a first approximation, may be described as planar. This is also clearly illustrated by the systems shown in Figure 2 and Figure S2 in the supplementary information, which plot also highlight the fact that the PA1234- $n$ systems with $n \geq 2$ exhibit quasi in-plane isotropic auxetic behaviour which tends to -1 as $n$ increases whilst the PA1245- $n$ systems are highly anisotropic and exhibit auxetic behaviour for loading in off-axis directions that approximately correspond to the $55^{\circ}$. Also, in general, the shapes of crystalline systems were similar to those of the monolayers, as were their in-plane Poisson's ratios and other properties as shown in Figure 3. In fact there is a linear relationship between the $c_{i j}$ of the crystalline systems and the respective monolayers as illustrated in Figure 4 and Table S1. Given this agreement between the properties of the monolayers and the crystals, the discussion in this paper will focus on the properties of the crystals.

\section{INSERT FIGURES 2 to 5}

Before proceeding any further it is important to note that as will be discussed in more detail below, there is an extremely strong relationship between the key architectural features in the nanosystems and their elastic response, with the elastic constants being reported here generally agreeing well to trends established in analytical models for these architectures 
described in the literature. For example, a common trend which is observed is that the stiffness, both in terms of Young's and shear moduli (see Figure 5) decreases as the length of the acetylene chains increases. This type of behaviour is to be expected and may be explained by looking at these systems from a purely mechanical point of view since these longer chains which are de facto behaving as nanobeams are easier to flex when compared to their shorter counterparts. Also, in the case of PA1245, the only systems which do not exhibit hexagonal symmetry, it can easily be identified from Figures 2 and S5 that the anisotropy in the stiffness may be mapped to the structures with the highest Young's moduli being exhibited in the directions of the acetylene chains. In the case of the Poisson's ratio for the tetrasubstituted auxetic systems, there is a general trend that as the length of the acetylene chains increases, the Poisson's ratio tends increasingly more to the value predicted by the analytical models for the respective idealised models, that is, -1 for the PA1234 systems mimicking the rotating triangles, and $\cot \left(\frac{\theta}{2}-\zeta\right) \cot \left(\frac{\theta}{2}+\zeta\right)$ where $\theta=\frac{3 \pi}{2}$ in the case of the PA1245 systems mimicking the wine-racks (see Figure 5) which were recently found to exhibit auxetic behaviour [63]. This trend may be explained by the fact that the systems with longer chains resemble the macrostructures they are meant to mimic to a much greater extent. Similar arguments can be made to the systems with conventional Poisson's ratios.

More specifically, for a given $n$, the minimised PA12345- $n$ and PA135- $n$ systems may both be described as honeycombs with hexagonal pores of equal size. This geometry, which has a hexagonal symmetry, renders these two sets of systems isotropic in-plane as clearly evident by all their respective mechanical property plots (see Figure S1 and Figure S3). However, the manner of construction of these two sets of systems is very different with the ligaments making up the honeycomb in PA135- $n$ systems being simply of acetylene chains whilst the ligaments of the PA12345- $n$ system are a much more complex truss system made from acetylene chains connecting via penta-substituted phenyl rings. This difference in the manner of construction results in very different magnitudes of the various mechanical properties with 
PA12345- $n$ variants being significantly more stiff, having a higher Young's moduli and higher shear moduli (see Figure 2). This increase in stiffness may be explained by the nanostructure and nanomechanics of the systems since whilst the PA135- $n$ systems $(n, n$ flexynes) may easily deform through flexure of the acetylene chains [42], flexure in the PA12345- $n$ systems is not easy to accomplish due to the triangulation that results in a trusslike system. Also interesting is the difference in the Poisson's ratio afforded by these systems, where the PA135- $n$ flexyne systems have their Poisson's ratio approaching +1 as $n$ increases whilst the PA12345- $n$ tend to have Poisson's ratios which are approximately half this value (see Figure S3). Here it should be noted that whilst it is beyond the scope of this work to provide a quantitative analysis of the deformations and nanomechanics of the different systems, it is to be expected that these two systems would not deform in an equivalent manner since the triangulation provides significant stiffening to the PA12345- $n$ system which is more than that expected from just the increase in density. This means that, from a structural point of view, these systems may be considered as more similar to the fully substituted PA123456- $n$ systems (i.e. graphyne, graphidyne, etc.) than the flexynes. In fact, it is interesting to note that the PA123456- $n$ and PA12345- $n$ systems have almost identical Poisson's ratio properties although their moduli differ in magnitude, as expected.

In the case of the PA1234- $n$ systems ( $n \geq 2)$, the Poisson's ratios tends to -1 as the length of the acetylene chain increases (see Figure S2). Such systems have already been studied in detail [9] and are known to exhibit auxetic behaviour due to a mechanism which involves relative rotation of the triangular units, a mechanism which is well known for its auxetic potential [29], [30]. Here it should be noted that simulations performed with the PCFF forcefield of the PA1234 system with $n=5$ suggest that the nanoscale deformations taking place in these networks are primarily due to flexure of the acetylene chains, which flexure still preserves the rotational symmetry of triangular unit with the consequence that the mechanism can still be described as 'rotation of semi-rigid triangular units' (see Figure 6). Similar 
behaviour has been observed when performing finite element modelling of equivalent systems made from beams [30], that is, this type of deformation is not restricted to the nanoscale. It is also worth highlighting that for any given $n$, these auxetic systems exhibit very low Young's moduli when compared to their non-auxetic counterparts studied here, which moduli are even lower than those of the less dense flexyne systems made from acetylene chains of equal length (see Table S1, Supporting information). This behaviour may be explained by the fact that these PA1234 systems deform in a manner where every acetylene chain in the system flexes in a manner that is highly amenable, with the result that the amount of energy required to accomplish such deformation is minimal. This further enhances the role of the rotation mechanism which results in the observed auxetic behaviour where the Poisson's ratio tends to the theoretical value of -1 .

\section{INSERT FIGURE 6}

Equally interesting are the PA1245 systems (see Figure 3 and 5), which are being studied for the first time, where it is being shown that these systems are capable of exhibiting very large negative Poisson's ratio in off-axis directions which in some cases are more negative than -3 . This feature has now been confirmed to be a characteristic of wine-rack like systems [61], and highlights the importance that one does not restrict the analysis to on-axis behaviour as discussed elsewhere.

All this is very significant since it has been shown that by changing the manner of substitution and/or length of the acetylene chain one can independently vary the pore-size, stiffness and/or the Poisson's ratio of these systems. This highlights the versatility of poly(phenylacetylene) networks which can be specifically designed to exhibit tailor-made mechanical or other properties. Here it should also be noted that these systems are expected to exhibit other useful properties which are either a result of the chemistry of the systems or their physics. For example, as a result of the extensive delocalisation of these systems, one would expect that polyphenyacetylenes would also be highly conductive polymers. Thus, the 
particular combination of this property with some specific set of mechanical properties is likely to broaden the practical importance of these networks.

Before we conclude it is important to note that the work presented here is based entirely on simulations performed using a static force-field based method. Although it was confirmed that the results obtained are force-field independent since the results being reported here obtained with the PCFF force-field are being confirmed by the Dreiding force-field, one may still argue that what is being reported here are just estimates of what is expected to happen if these systems would be synthesised and tested. Obviously, irrespective of how complex and intricate a model may be, any predictions obtained from it would remain estimates. However, one may equally argue that the main strength of this work is the comparison of the trends and it is more than likely that any qualitative conclusions that are being made are valid. This is justified by the fact that the trends which are being reported are in line with what one would expect through a geometric analysis of the systems. For example, the isotropic nature of the results obtained for the PA135, PA1234, PA12345 and PA123456 systems is a consequence of the crystal symmetry of the systems (a geometric effect) and the fact that the calculated mechanical properties are in accordance with this symmetry confirms validity of this work. Nevertheless, it is only through experimental work that the predictions being made here can be finally confirmed and it is hoped that this work will provide an impetus to experimentalists to synthesise and test systems based on the work presented here.

It is also equally important to highlight the fact that although the crystal systems and the monolayer systems exhibit similar trends, large scale effects that could be present in monolayer systems have not been considered. This is primarily due to the fact that throughout all simulations, a small unit cell size was used which cannot encompass macroscale effects. In particular, it is well known that in large sheets of graphene in which defects have been introduced, there is a tendency for the formation of ripples, which may themselves give rise to interesting properties including auxeticity amongst others [11], [62]. Obviously, such an 
effect is not possible to reproduce on a small scale where the unit cell only contains a few tens of atoms. Although one might argue that overlooking such effects may raise doubt on the validity of the results presented in this work, it should be noted that the effects described in this work are still likely to be present in a more localised manner on a nanoscale rather than on a macroscopic scale. Nevertheless, should one wish to estimate the properties that monolayers exhibit on the large scale, it would be more appropriate to carry out molecular dynamics simulations of considerably larger systems since such techniques are likely to be more appropriate for simulation of the macroscale behaviour of such membranes. In addition, given that the simulations were of a static nature, molecular dynamics could also provide insights into how the mechanical properties of these networks change with temperature. One may argue that the presence of acetylene chains lends the networks a degree of flexibility so that an increase in temperature is likely to cause a degree of rippling in the networks, an effect which has been reported in graphyne and graphdiyne [62]. This rippling, modifies the macro-scale geometry of the sheet so that other mechanisms may come into effect when loads are applied to the system. More specifically, one would expect that 'flattening' of the sheet is the dominant mechanism at smaller strains, which is likely to be characterised by relatively low Young's moduli when compared to the flatter counterparts modelled here. Nonetheless, the mechanisms leading to the properties reported here are still very likely to operate although at higher strains.

It should finally be noted that although the systems described here are yet to be synthesised, it is more than likely that what has been reported for very specific poly(phenylacetylene) networks would also be applicable to other systems which have similar architectural features. Given the fact that auxetics are now being increasingly sought after, it is hoped that the present work will be of benefit to the scientific community and bring the subject of negative Poisson's ratios, including some of the earlier and fundamental work in this field [64-68], to the limelight. 


\section{Conclusion}

This work has explored the mechanical properties of a number of molecular systems based on poly(phenylacetylene) networks, through a force-field based method. It has been shown that by varying the type of substitution on the phenyl ring and the length of the acetylene chain, one may be able to control the mechanical properties of the system, namely stiffness, linear compressibility and Poisson's ratio. In particular, the results suggest that polyphenylacetlenes made from tri, penta and hexa substituted phenyls (PA135- $n$, PA12345- $n$ and PA123456- $n$ ) exhibit a positive isotropic Poisson's ratio. In contrast, those made from tetra substituted phenyls (PA1234- $n$ and PA1245- $n$ ) can be designed to exhibit negative Poisson's ratios that become increasingly negative with higher $n$-values. In addition, the PA1234- $n$ networks, which mimic the rotating triangles model exhibit an almost isotropic behaviour which tends to -1 with increasing $n$ whilst the PA1245- $n$, which mimic the wine-rack structure, have a negative on-axis linear compressibility, and very highly negative Poisson's ratio off-axis. It was also found that PA12345- $n$ and PA135- $n$ have the same pore sizes but PA12345- $n$ are much stiffer in terms of Young's and shear moduli, and lower linear compressibility, which behaviour is expected from a geometric perspective. All this is very significant as it highlights the possibility of tailoring these extremely versatile networks to exhibit particular mechanical behaviour.

\section{Acknowledgements}

The research work disclosed in this publication is funded by the ENDEAVOUR Scholarship Scheme (Malta). The scholarship may be part-financed by the European Union - European Social Fund (ESF) under Operational Programme II - Cohesion Policy 2014-2020, “Investing 
in human capital to create more opportunities and promote the well being of society". JNG acknowledges the support of the University of Malta research grant.

\section{References:}

[1] K. E. Evans, M. A. Nkansah, I. J. Hutchinson, and S. C. Rogers, Molecular Network Design, Nature 353, 12-125 (1991).

[2] J. N. Grima, R. Jackson, A. Alderson, and K. E. Evans, Do Zeolites Have Negative Poisson's Ratios?, Adv. Mater. 12, 1912-1918 (2000).

[3] M. Siddorn, F.-X. Coudert, K. E. Evans, and A. Marmier, A systematic typology for negative Poisson's ratio materials and the prediction of complete auxeticity in pure silica zeolite JST, Phys. Chem. Chem. 17, 17927-17933 (2015).

[4] F. Nazaré and A. Alderson, Models for the prediction of Poisson's ratio in the ' $\alpha$ cristobalite' tetrahedral framework, Phys. status solidi 252, 1465-1478 (2015).

[5] R. Gatt et al., Hierarchical Auxetic Mechanical Metamaterials, Sci. Rep. 5, 8395 (2015).

[6] K. M. Azzopardi, J. P. Brincat, J. N. Grima, and R. Gatt, Anomalous elastic properties in stishovite, $R S C A d v$. 5, 8974-8980 (2015).

[7] C. He, P. Liu, and A. C. Griffin, Toward Negative Poisson Ratio Polymers through Molecular Design, Macromolecules 31, 3145-3147 (1998).

[8] C. He, P. Liu, P. J. McMullan, and A. C. Griffin, Toward molecular auxetics: Main chain liquid crystalline polymers consisting of laterally attached para-quaterphenyls, Phys. status solidi 242, 576-584 (2005).

[9] J. N. Grima and K. E. Evans, Self expanding molecular networks, Chem. Commun. 
$1531-1532(2000)$.

[10] J. N. Grima, C. Zerafa, and J.-P. Brincat, Development of novel poly(phenylacetylene) network polymers and their mechanical behaviour, Phys. status solidi 251, 375-382 (2014).

[11] J. N. Grima et al., Tailoring Graphene to Achieve Negative Poisson's Ratio Properties, Adv. Mater. 27, 1455-1459 (2015).

[12] R. H. Baughman and D. S. Galvão, Crystalline networks with unusual predicted mechanical and thermal properties, Nature 365, 735-737 (1993).

[13] R. H. Baughman, J. M. Shacklette, A. A. Zakhidov, and S. Stafström, Negative Poisson's ratios as a common feature of cubic metals, Nature 392, 362-365 (1998).

[14] A. C. Brańka, D. M. Heyes, and K. W. Wojciechowski, Auxeticity of cubic materials, Phys. status solidi 246, 2063-2071 (2009).

[15] R. Lakes, Foam Structures with a Negative Poisson's Ratio, Science (80-. ). 235, 1038-1040 (1987).

[16] N. Chan and K. E. Evans, Fabrication methods for auxetic foams, J. Mater. Sci. 32, 5945-5953 (1997).

[17] F. Scarpa, P. Pastorino, A. Garelli, S. Patsias, and M. Ruzzene, Auxetic compliant flexible PU foams: Static and dynamic properties, Phys. Status Solidi Basic Res. 242, 681-694 (2005).

[18] J. N. Grima, D. Attard, R. Gatt, and R. N. Cassar, A Novel Process for the Manufacture of Auxetic Foams and for Their re-Conversion to Conventional Form, Adv. Eng. Mater. 11, 533-535 (2009).

[19] A. Bezazi and F. Scarpa, Tensile fatigue of conventional and negative Poisson's ratio open cell PU foams, Int. J. Fatigue 31, 488-494 (2009). 
[20] S. A. McDonald, G. Dedreuil-Monet, Y. T. Yao, A. Alderson, and P. J. Withers, In situ 3D X-ray microtomography study comparing auxetic and non-auxetic polymeric foams under tension, Phys. status solidi 248, 45-51 (2011).

[21] B. D. Caddock and K. E. Evans, Microporous materials with negative Poisson's ratios. I. Microstructure and mechanical properties, J. Phys. D. Appl. Phys. 22, 1877-1882 (1989).

[22] K. L. Alderson and K. E. Evans, The fabrication of microporous polyethylene having a negative Poisson's ratio, Polymer (Guildf). 33, 4435-4438 (1992).

[23] R. S. Webber, K. L. Alderson, and K. E. Evans, Novel variations in the microstructure of the auxetic microporous ultra-high molecular weight polyethylene. Part 1: Processing and microstructure, Polym. Eng. Sci. 40, 1894-1905 (2000).

[24] K. L. Alderson, R. S. Webber, A. P. Kettle, and K. E. Evans, Novel fabrication route for auxetic polyethylene. Part 1. Processing and microstructure, Polym. Eng. Sci. 45, $568-578(2005)$.

[25] L. J. Gibson, M. F. Ashby, G. S. Schajer, and C. I. Robertson, The Mechanics of TwoDimensional Cellular Materials, Proc. R. Soc. A Math. Phys. Eng. Sci. 382, 25-42 (1982).

[26] A. Bezazi, F. Scarpa, and C. Remillat, A novel centresymmetric honeycomb composite structure, Compos. Struct. 71, 356-364 (2005).

[27] R. Cauchi, D. Attard, and J. N. Grima, On the mechanical properties of centrosymmetric honeycombs with T-shaped joints, Phys. status solidi 250, 2002-2011 (2013).

[28] J. N. Grima and K. E. Evans, Auxetic behavior from rotating squares, J. Mater. Sci. Lett. 19, 1563-1565 (2000). 
[29] J. N. Grima and K. E. Evans, Auxetic behavior from rotating triangles, J. Mater. Sci. 41, 3193-3196 (2006).

[30] J. N. Grima et al., On the auxetic properties of generic rotating rigid triangles, Proc. R. Soc. A 468, 810-830 (2012).

[31] J. N. Grima, P.-S. Farrugia, R. Gatt, and D. Attard, On the auxetic properties of rotating rhombi and parallelograms: A preliminary investigation, Phys. status solidi 245, 521-529 (2008).

[32] D. Attard and J. N. Grima, A three-dimensional rotating rigid units network exhibiting negative Poisson's ratios, Phys. status solidi 249, 1330-1338 (2012).

[33] T. Bückmann, R. Schittny, M. Thiel, M. Kadic, G. W. Milton, and M. Wegener, On three-dimensional dilational elastic metamaterials, New J. Phys. 16, 33032 (2014).

[34] G. W. Milton, New examples of three-dimensional dilational materials, Phys. status solidi 252, 1426-1430 (2015).

[35] K. V. Tretiakov and K. W. Wojciechowski, Monte Carlo simulation of twodimensional hard body systems with extreme values of the Poisson's ratio, Phys. status solidi 242, 730-741 (2005).

[36] M. Bilski and K. W. Wojciechowski, Tailoring Poisson's ratio by introducing auxetic layers, Phys. status solidi 253, 1318-1323 (2016).

[37] D. Prall and R. S. Lakes, Properties of a chiral honeycomb with a poisson's ratio of 1, Int. J. Mech. Sci. 39, 305-314 (1997).

[38] J. N. Grima, R. Gatt, and P.-S. Farrugia, On the properties of auxetic meta-tetrachiral structures, Phys. status solidi 245, 511-520 (2008).

[39] A. Alderson et al., Elastic constants of 3-, 4- and 6-connected chiral and anti-chiral honeycombs subject to uniaxial in-plane loading, Compos. Sci. Technol. 70, 1042- 
1048 (2010).

[40] A. A. Pozniak and K. W. Wojciechowski, Poisson's ratio of rectangular anti-chiral structures with size dispersion of circular nodes, Phys. status solidi 251, 367-374 (2014).

[41] M. R. Sloan, J. R. Wright, and K. E. Evans, The helical auxetic yarn - A novel structure for composites and textiles; geometry, manufacture and mechanical properties, Mech. Mater. 43, 476-486 (2011).

[42] M. A. Nkansah, K. E. Evans, and I. J. Hutchinson, Modelling the mechanical properties of an auxetic molecular network, Model. Simul. Mater. Sci. Eng. 2, 337-352 (1994).

[43] K. E. Evans, A. Alderson, and F. R. Christian, Auxetic two-dimensional polymer networks. An example of tailoring geometry for specific mechanical properties, $J$. Chem. Soc. Faraday Trans. 91, 2671 (1995).

[44] Z.-G. Shao and Z.-L. Sun, Optical properties of alpha-, beta- gamma-, and 6,6,12graphyne structures: First-principle calculations, Phys. E - Low-Dimensional Syst. Nanostructures 74, 438-442 (2015).

[45] Y. Li, L. Xu, H. Liu, and Y. Li, Graphdiyne and graphyne: from theoretical predictions to practical construction, Chem. Soc. Rev. 43, 2572-2586 (2014).

[46] Z.-Z. Lin, Q. Wei, and X. Zhu, Modulating the electronic properties of graphdiyne nanoribbons, Carbon N. Y. 66, 504-510 (2014).

[47] S. W. Cranford and M. J. Buehler, Mechanical properties of graphyne, Carbon N. Y. 49, 4111-4121 (2011).

[48] Y. Yang and X. Xu, Mechanical properties of graphyne and its family - A molecular dynamics investigation, Comput. Mater. Sci. 61, 83-88 (2012). 
[49] J. Kang, J. Li, F. Wu, S.-S. Li, and J.-B. Xia, Elastic, Electronic, and Optical Properties of Two-Dimensional Graphyne Sheet, J. Phys. Chem. C 115, 20466-20470 (2011).

[50] Q. Peng, W. Ji, and S. De, Mechanical properties of graphyne monolayers: a firstprinciples study, Phys. Chem. Chem. Phys. 14, 13385 (2012).

[51] Y. Pei, Mechanical properties of graphdiyne sheet, Phys. B Condens. Matter 407, 4436-4439 (2012).

[52] Y. Li, L. Xu, H. Liu, and Y. Li, Graphdiyne and graphyne: from theoretical predictions to practical construction, Chem. Soc. Rev. Chem. Soc. Rev 43, 2572-2586 (2572).

[53] J. N. Grima, D. Attard, R. Caruana-Gauci, and R. Gatt, Negative linear compressibility of hexagonal honeycombs and related systems, Scr. Mater. 65, 565-568 (2011).

[54] H. Sun, S. J. Mumby, J. R. Maple, and A. T. Hagler, An ab Initio CFF93 All-Atom Force Field for Polycarbonates, J. Am. Chem. Soc. 116, 2978-2987 (1994).

[55] H. Sun, Ab initio calculations and force field development for computer simulation of polysilanes, Macromolecules 28, 701-712 (1995).

[56] H. Sun and D. Rigby, Polysiloxanes: ab initio force field and structural, conformational and thermophysical properties, Spectrochim. Acta Part A Mol. Biomol. Spectrosc. 53, 1301-1323 (1997).

[57] P. P. Ewald, Die Berechnung optischer und elektrostatischer Gitterpotentiale, Ann. Phys. 369, 253-287 (1921).

[58] J. F. Nye, Physical properties of crystals: Their representation by tensor and matrices. 1957.

[59] S. L. Mayo, B. D. Olafson, and W. A. Goddard Iii, DREIDING: A Generic Force Field for Molecular Simulations, J . Phys. Chem 94, 8897-8909 (1990). 
[60] A. K. Rappe and W. A. Goddard III, Charge Equilibration for Molecular Dynamics Simulations, J. Phys. Chem. 95, 3358-3363 (1991).

[61] H. Qin, Y. Sun, J. Z. Liu, M. Li, and Y. Liu, Negative Poisson's ratio in rippled graphene, Nanoscale 98, 13113 (2017).

[62] A. L. Ivanovskii, Graphynes and graphdyines, Prog. Solid State Chem. 41, 1-19 (2013)

[63] R. Caruana-Gauci, E.P. Degabriele, D. Attard and J.N. Grima, Auxetic Metamaterials Inspired from Wine-Racks, J. Mater. Sci. (Submitted).

[64] K. W. Wojciechowski, Constant thermodynamic tension Monte Carlo studies of elastic properties of a two-dimensional system of hard cyclic hexamers, Mol. Phys. 61, 12471258 (1987).

[65] K. W. Wojciechowski, Two-dimensional isotropic system with a negative poisson ratio, Phys. Lett. A 137, 60-64 (1989).

[66] K. W. Wojciechowski, Remarks on "Poisson Ratio beyond the Limits of the Elasticity Theory,” J. Phys. Soc. Japan 72, 1819-1820 (2003).

[67] K. W. Wojciechowski, Non-chiral, molecular model of negative Poisson ratio in two dimensions, J. Phys. A. Math. Gen. 36, 11765-11778 (2003).

[68] K. W. Wojciechowski, Monte Carlo Simulations of Model Particles Forming Phases of Negative Poisson Ratio, , in Properties and Applications of Nanocrystalline Alloys from Amorphous Precursors, Berlin/Heidelberg: Springer-Verlag, 2005, 241-252 . 


\section{Figures}

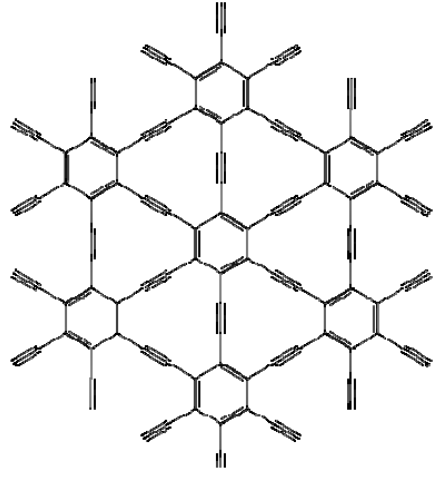

(a)

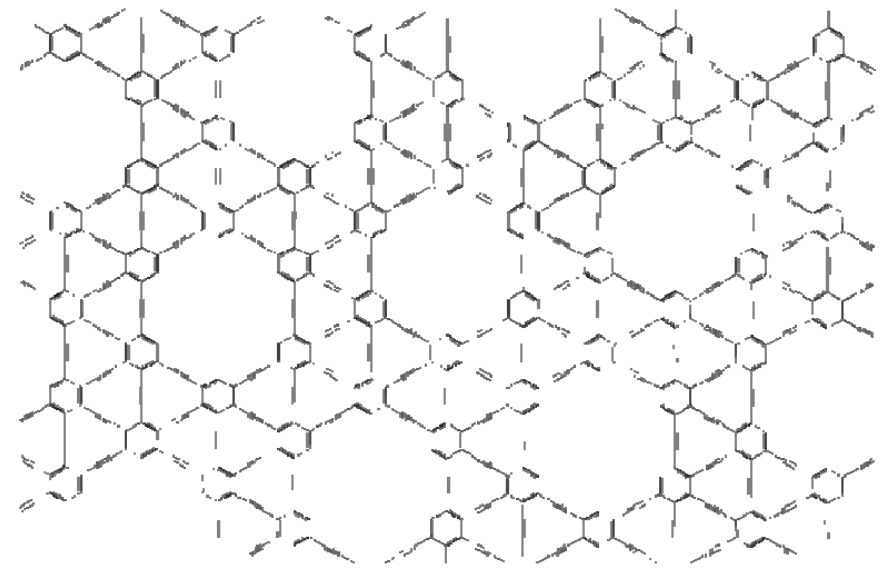

(b)

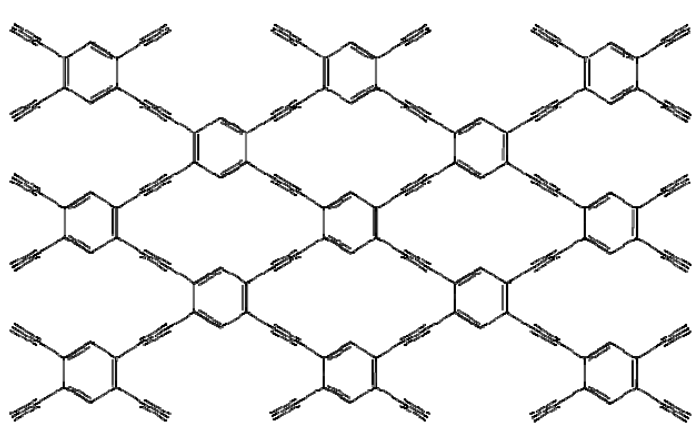

(c)

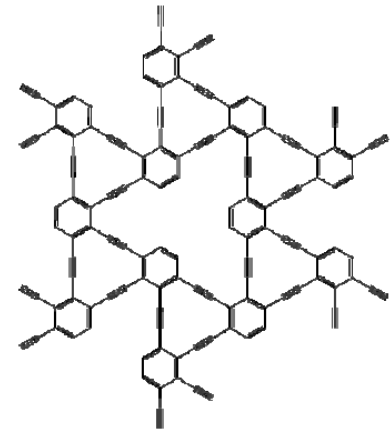

(d)

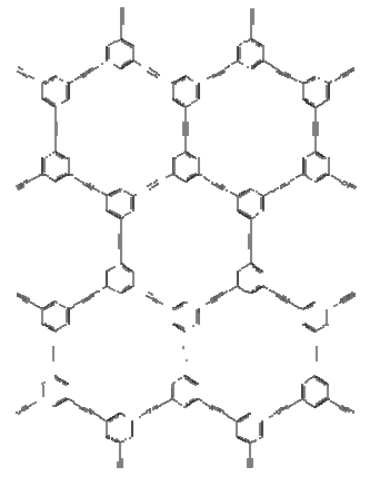

(e)

Figure 1: Schematic diagrams of the systems modelled having $n$ triple bonds per acetylene chains: (a) the fully substituted systems, henceforth referred to as PA123456$n$; (b) the penta substituted systems, henceforth referred to as PA12345- $n$ [53]; (c) the $1,2,4,5$ tetra-substituted networks which resemble the wine-racks, henceforth referred to as PA1245-n; (d) the 1,2,3,4 tetra-substituted networks which correspond to the polytriangles-n-yne [9], henceforth referred to as PA1245- $n$; and (e) the 1,3,5 trisubstituted networks which correspond to the regular $n, n$-flexynes [42] henceforth referred to as PA135- $n$. 


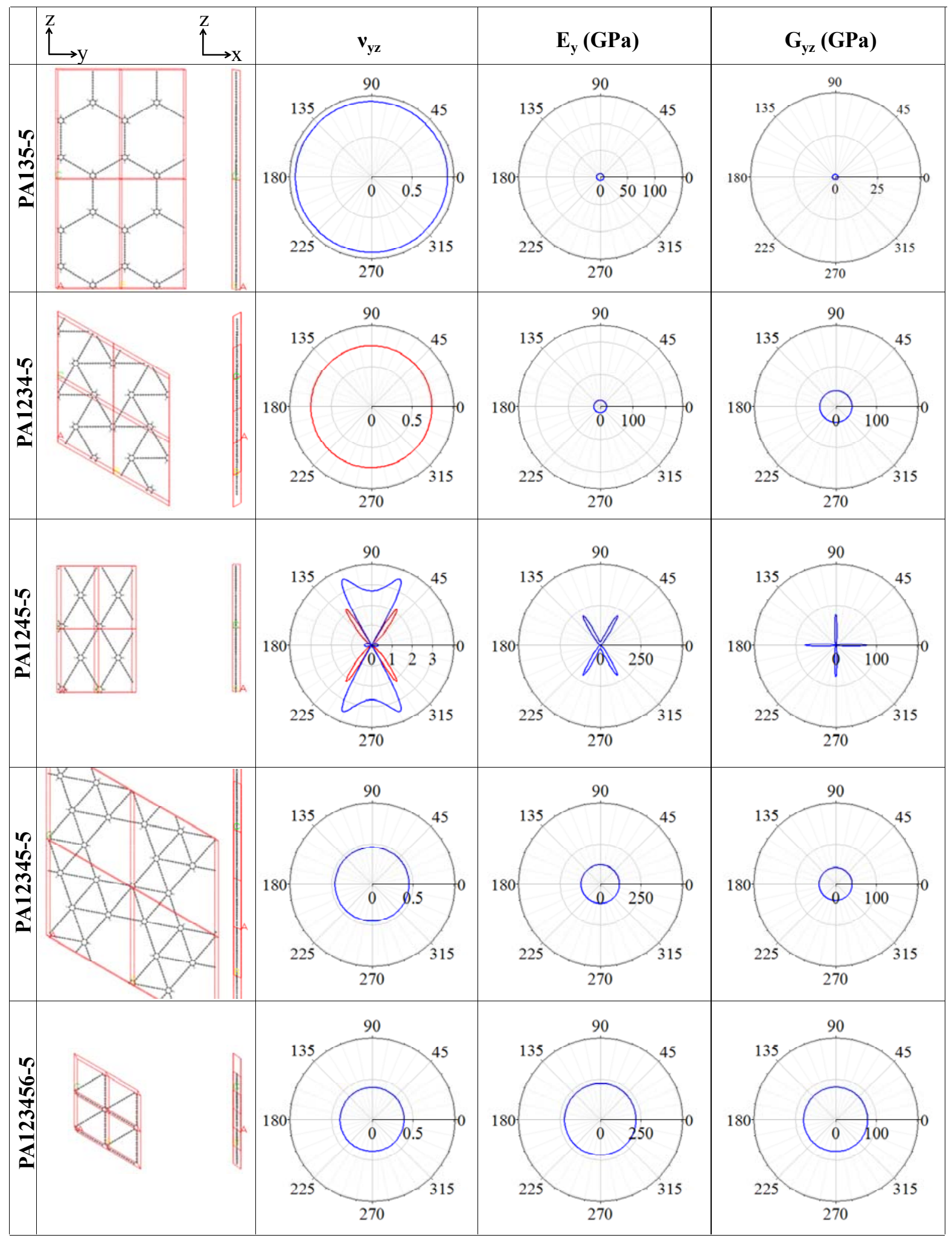

Figure 2: Images of the crystalline systems where $n=5$ and their off-axis plots in the yzplane (the plane of the networks) of the Poisson's ratio $v_{\mathrm{yz}}$ Young's modulus $\mathbf{E}_{\mathrm{y}}$ and shear modulus in the planes of the networks as simulated by the PCFF force-field. Negative Poisson's ratios are shown in red while positive Poisson's ratios are shown in blue. It is clear that PA135, PA12345 and PA123456 show no negative Poisson's ratios. PA1234 shows an isotropic negative Poisson's ratio of $c$. 0.75 whereas PA1245 shows anisotropic behaviour with negative Poisson's ratios for loading off-axis at an angle nearly parallel to one of the acetylene chains. 


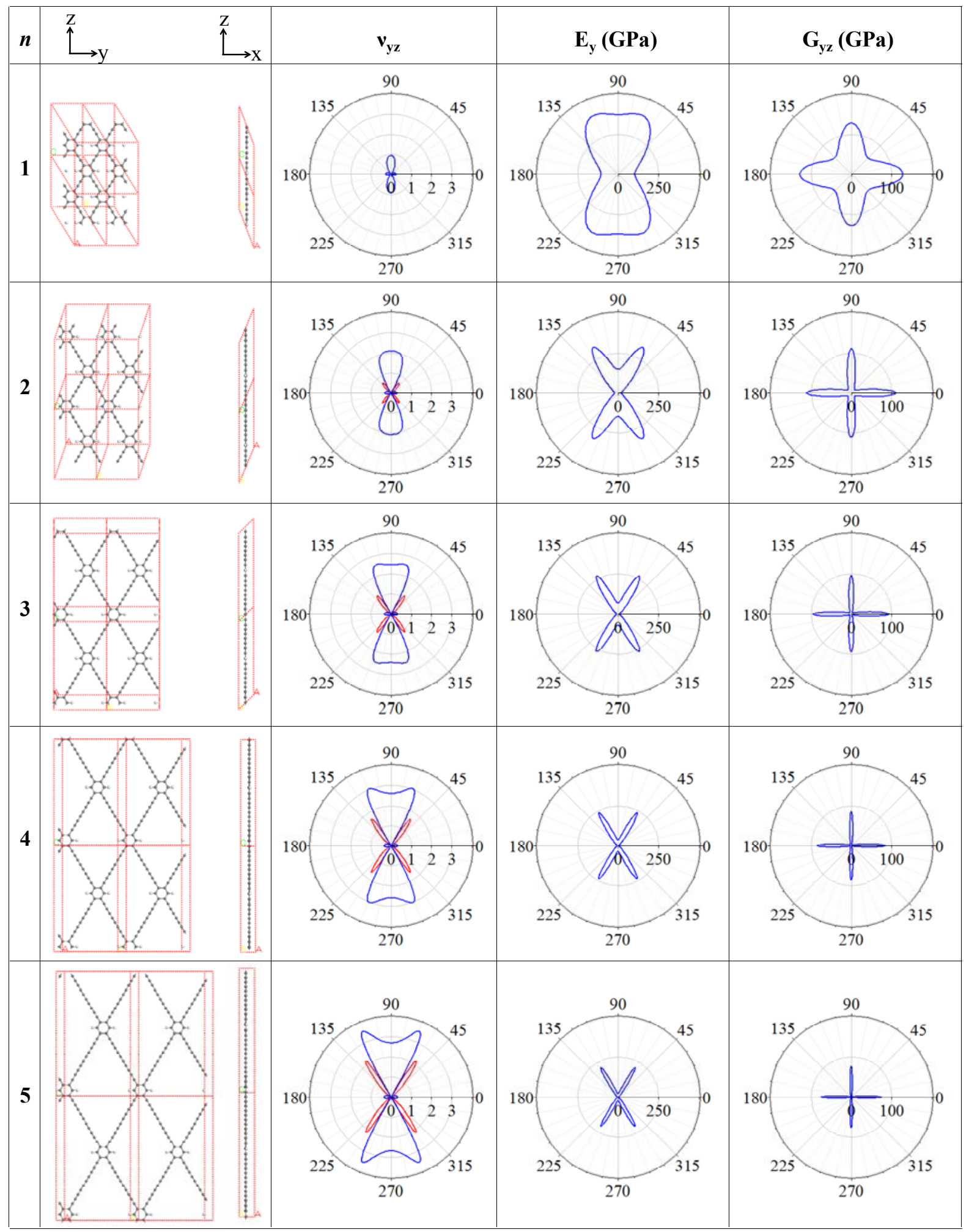

Figure 3: Images of the novel crystalline systems: PA1245-n, together with their off-axis plots of the Poisson's ratio, $v_{y z}$, Young's modulus, $E_{y}$, and shear modulus, $G_{y z}$, in the planes of the networks as simulated by the PCFF force-field. 


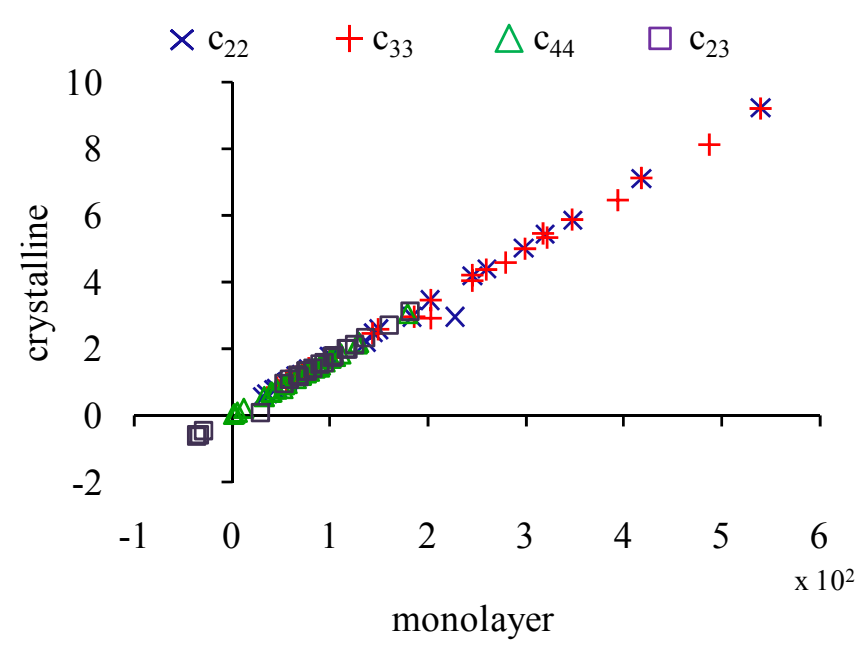

Figure 4: A plot of the $c_{i j}$ of the crystalline systems against the $c_{i j}$ of the respective monolayers. The units for the $c_{i j}$ coefficients are in GPa. 


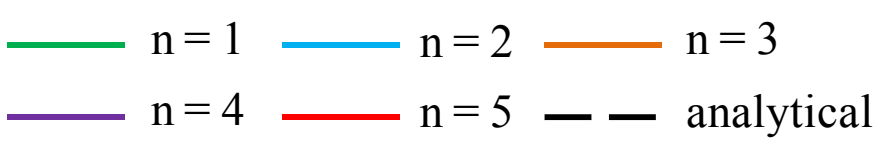

(a) $\mathbf{v}_{23}$

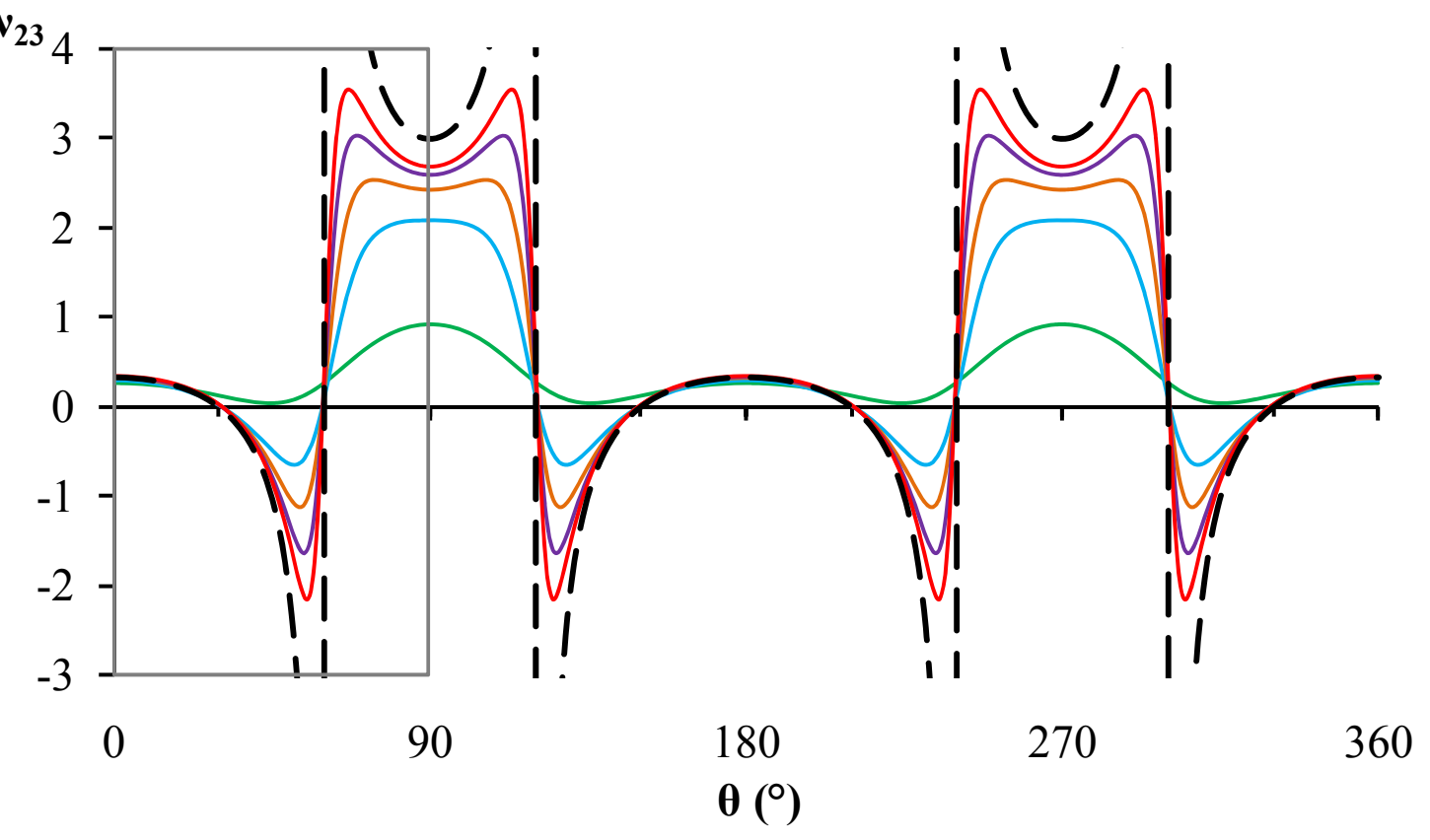

(b) $\mathbf{v}_{\mathbf{2 3}}$

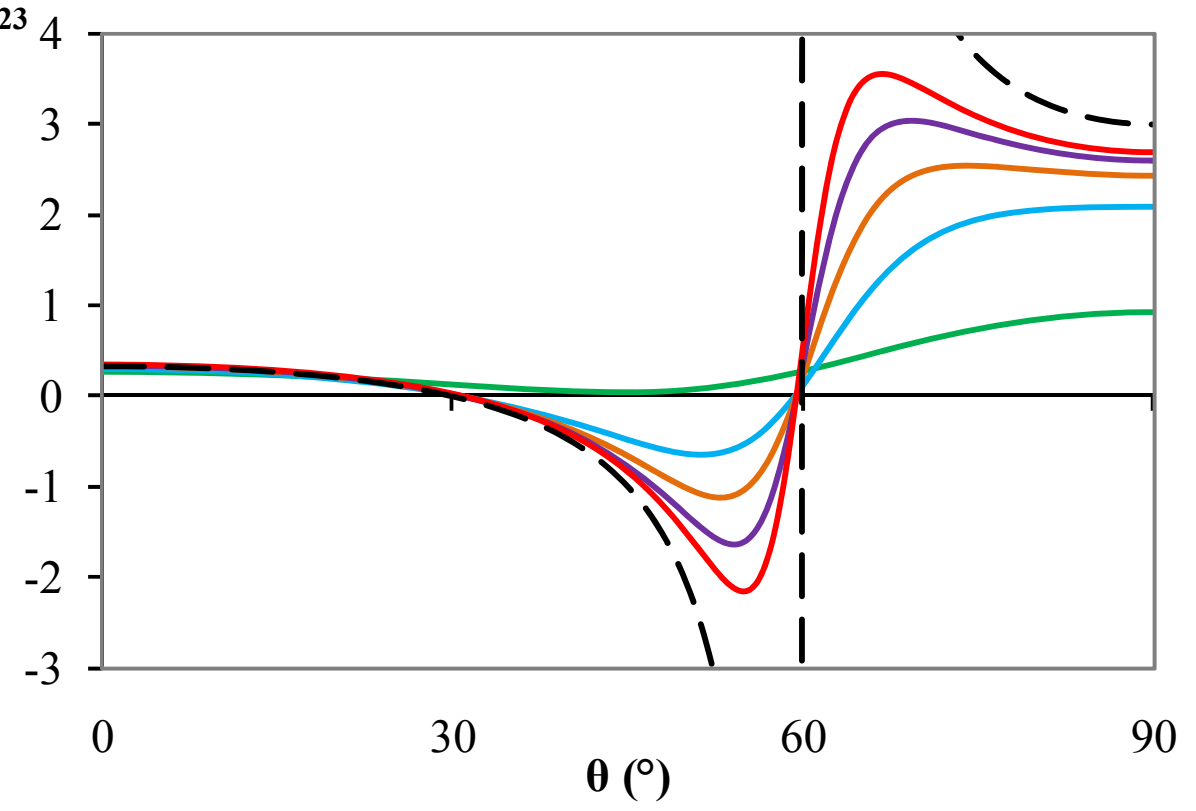

Figure 5:(a) An alternative representation of the off-axis plot of the Poisson's ratio in the $y z$ plane against the angle of rotation, $\theta$, for the PA1245- $n$ crystalline systems where they are compared to the analytical model [63] (b) A close-up at the Poisson's ratio in the range of $0^{\circ}$ to $90^{\circ}$ indicates that when $n=1$, the system shows a positive Poisson's ratio, while for systems $n=2,3,4,5$ the range of angles at which negative Poisson's ratio occurs is between $30.74^{\circ}$ and $59.23^{\circ}$. This plot also shows that as $n$ increases, the behaviour of the system mimics increasingly better the behaviour of the idealised winerack model (which was recently found to be auxetic [61]). 


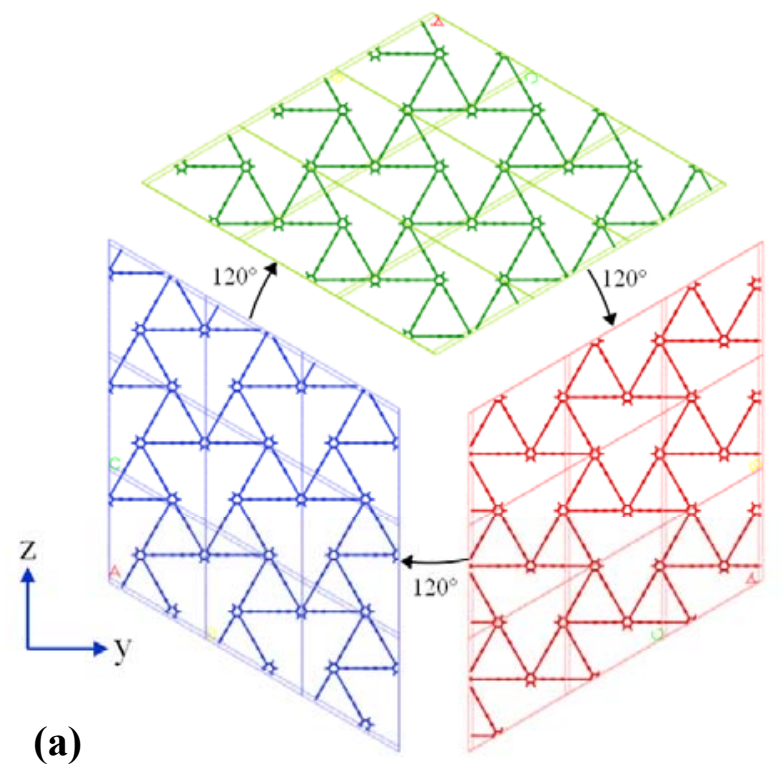

(a)
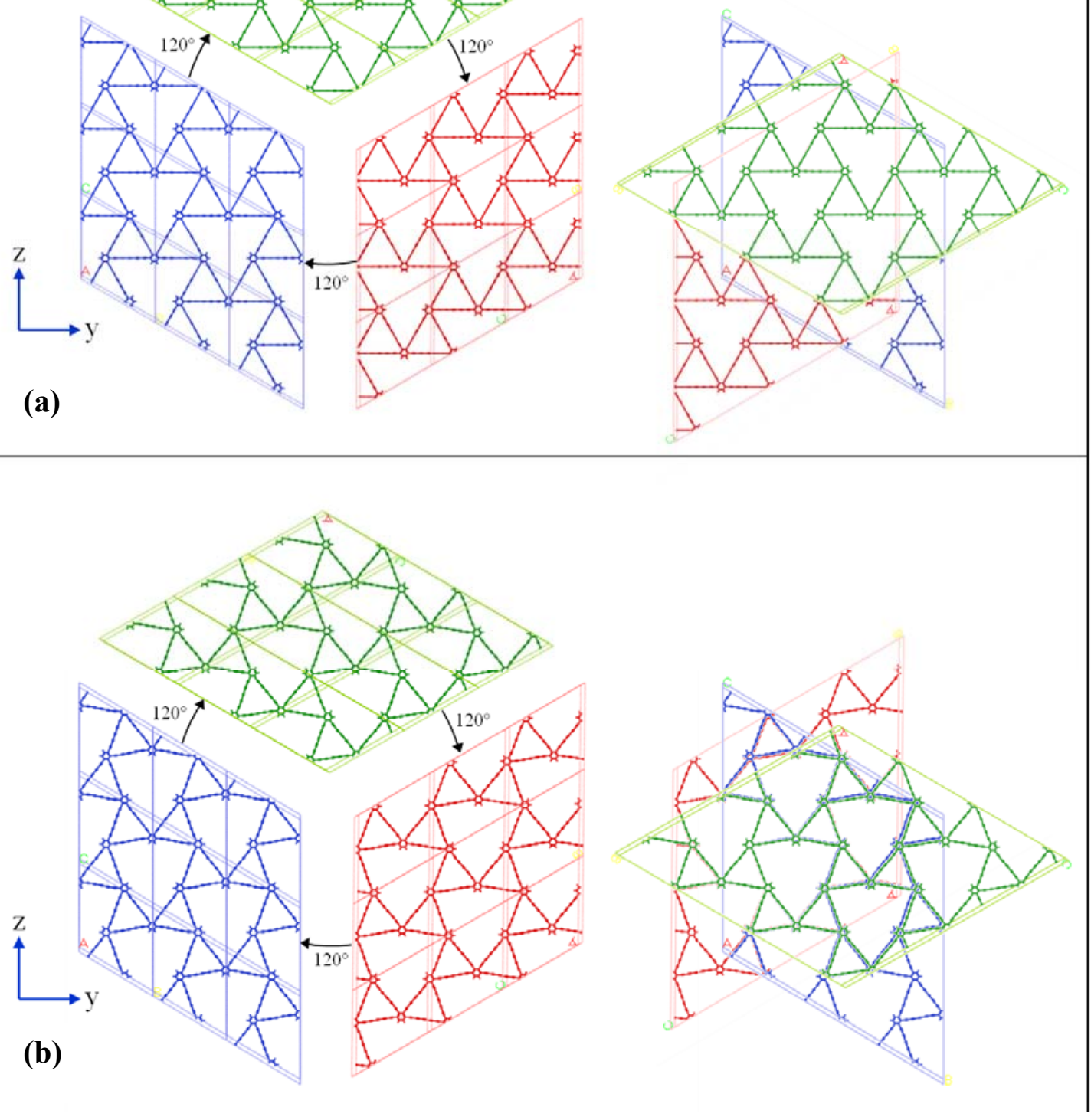

Figure 6: Consecutive $120^{\circ}$ rotation for the PA1234-5 network under a uniaxial stress of (a) 0 GPa and (b) 2 GPa, showing the preservation of the three-fold rotational symmetry characteristic of the idealised rotating rigid triangles system. 
SUPPLEMENTARY INFORMATION

\begin{tabular}{|c|c|c|c|c|c|c|c|c|c|}
\hline \multirow{2}{*}{ PA } & \multirow{2}{*}{$n$} & \multicolumn{4}{|c|}{ crystalline systems } & \multicolumn{4}{|c|}{ monolayer systems } \\
\hline & & 22 & 33 & 44 & 23 & 22 & 33 & 44 & 23 \\
\hline \multirow{5}{*}{$\stackrel{n}{2}$} & 1 & 143.698 & 143.587 & 33.116 & 77.394 & 2.466 & 2.466 & 0.569 & 1.328 \\
\hline & 2 & 99.419 & 99.387 & 12.208 & 74.998 & 1.753 & 1.753 & 0.216 & 1.321 \\
\hline & 3 & 78.141 & 78.080 & 5.704 & 66.704 & 1.392 & 1.392 & 0.101 & 1.190 \\
\hline & 4 & 65.136 & 65.177 & 3.048 & 59.065 & 1.166 & 1.166 & 0.055 & 1.057 \\
\hline & 5 & 56.034 & 56.023 & 1.820 & 52.391 & 1.009 & 1.009 & 0.033 & 0.944 \\
\hline & & & & & & & & & \\
\hline \multirow{5}{*}{$\stackrel{ \pm}{\beth}$} & 1 & 227.743 & 203.145 & 89.267 & 28.983 & 2.940 & 2.884 & 1.456 & 0.088 \\
\hline & 2 & 101.614 & 101.948 & 65.656 & -29.797 & 1.733 & 1.733 & 1.110 & -0.488 \\
\hline & 3 & 71.167 & 71.062 & 53.555 & -36.016 & 1.229 & 1.229 & 0.921 & -0.612 \\
\hline & 4 & 55.130 & 55.365 & 45.662 & -36.013 & 0.962 & 0.962 & 0.791 & -0.621 \\
\hline & 5 & 45.505 & 45.733 & 39.886 & -34.074 & 0.799 & 0.799 & 0.696 & -0.593 \\
\hline & & & & & & & & & \\
\hline \multirow{5}{*}{$\stackrel{\mathscr{N}}{\simeq}$} & 1 & 136.600 & 486.970 & 127.726 & 125.617 & 2.198 & 8.100 & 2.146 & 2.100 \\
\hline & 2 & 56.678 & 393.577 & 111.042 & 117.989 & 0.973 & 6.442 & 1.851 & 1.978 \\
\hline & 3 & 42.948 & 321.100 & 94.990 & 103.914 & 0.754 & 5.332 & 1.612 & 1.775 \\
\hline & 4 & 36.006 & 278.793 & 84.696 & 93.140 & 0.629 & 4.578 & 1.427 & 1.576 \\
\hline & 5 & 31.097 & 244.878 & 75.805 & 83.128 & 0.544 & 4.020 & 1.279 & 1.409 \\
\hline & & & & & & & & & \\
\hline \multirow{5}{*}{$\stackrel{n}{\stackrel{n}{\beth}}$} & 1 & 319.817 & 317.636 & 101.045 & 117.070 & 5.441 & 5.441 & 1.721 & 2.000 \\
\hline & 2 & 245.270 & 245.604 & 70.853 & 103.739 & 4.183 & 4.184 & 1.208 & 1.768 \\
\hline & 3 & 201.968 & 202.154 & 56.291 & 89.477 & 3.445 & 3.445 & 0.960 & 1.525 \\
\hline & 4 & 184.033 & 185.467 & 52.158 & 75.075 & 2.941 & 2.943 & 0.808 & 1.324 \\
\hline & 5 & 149.406 & 149.589 & 40.818 & 67.857 & 2.577 & 2.577 & 0.704 & 1.169 \\
\hline & & & & & & & & & \\
\hline \multirow{5}{*}{ 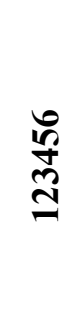 } & 1 & 539.487 & 539.492 & 179.049 & 181.393 & 9.215 & 9.215 & 3.060 & 3.094 \\
\hline & 2 & 417.937 & 418.406 & 129.075 & 159.988 & 7.100 & 7.100 & 2.193 & 2.714 \\
\hline & 3 & 348.276 & 347.593 & 105.519 & 136.923 & 5.854 & 5.854 & 1.775 & 2.304 \\
\hline & 4 & 297.502 & 299.137 & 90.335 & 117.670 & 5.006 & 5.006 & 1.515 & 1.976 \\
\hline & 5 & 260.437 & 259.835 & 79.025 & 102.070 & 4.381 & 4.381 & 1.330 & 1.720 \\
\hline
\end{tabular}

Table S1: The elastic constants, $c_{i j}$ (GPa) for the in-plane mechanical properties of the various systems studied. 


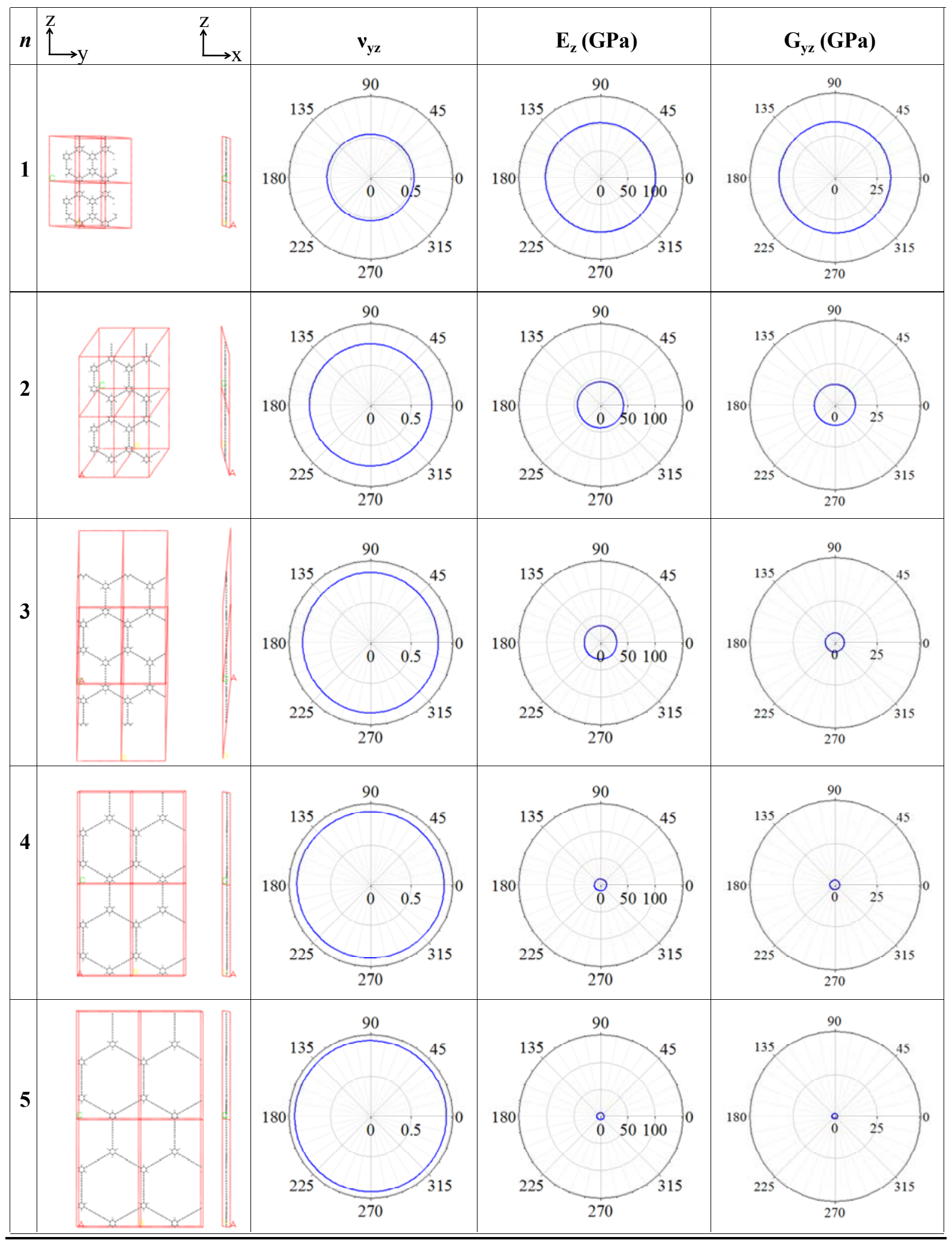

Figure S1: Structures and off-axis mechanical properties of the crystalline PA135n systems as simulated by the PCFF force-field. 


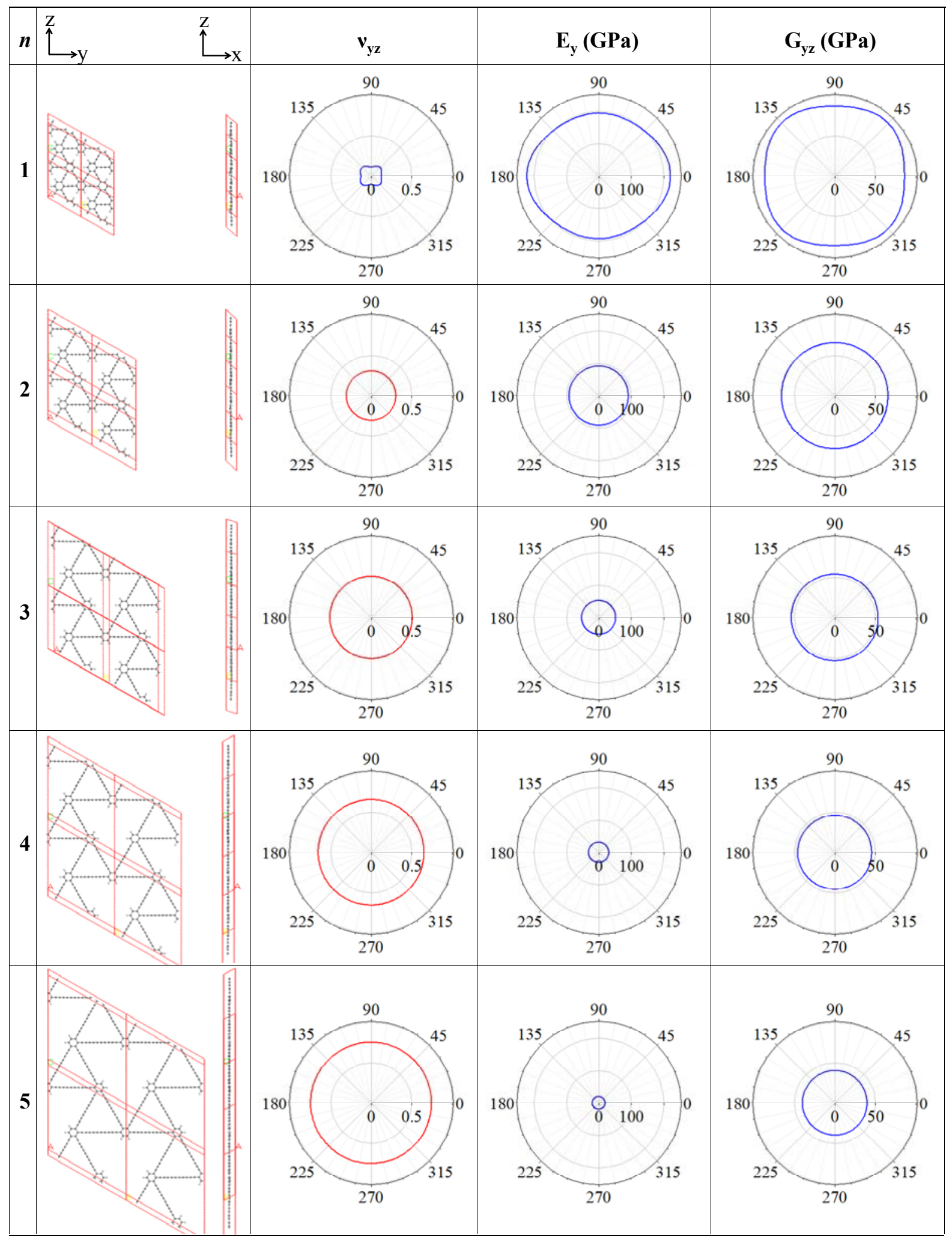

Figure S2: Structures and off-axis mechanical properties of the crystalline PA1234n systems as simulated by the PCFF force-field. 


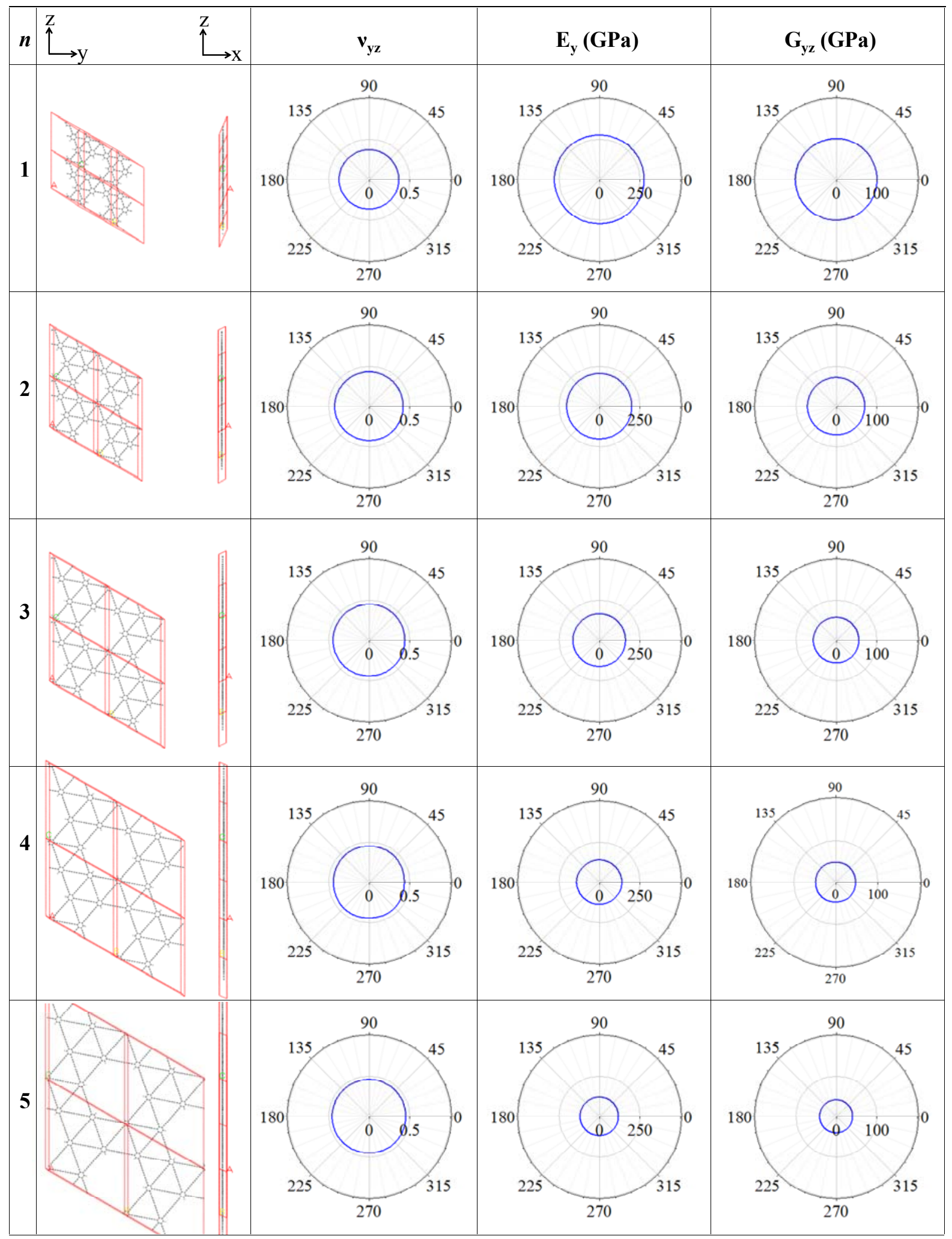

Figure S3: Structures and off-axis mechanical properties of the crystalline PA12345n systems as simulated by the PCFF force-field. 


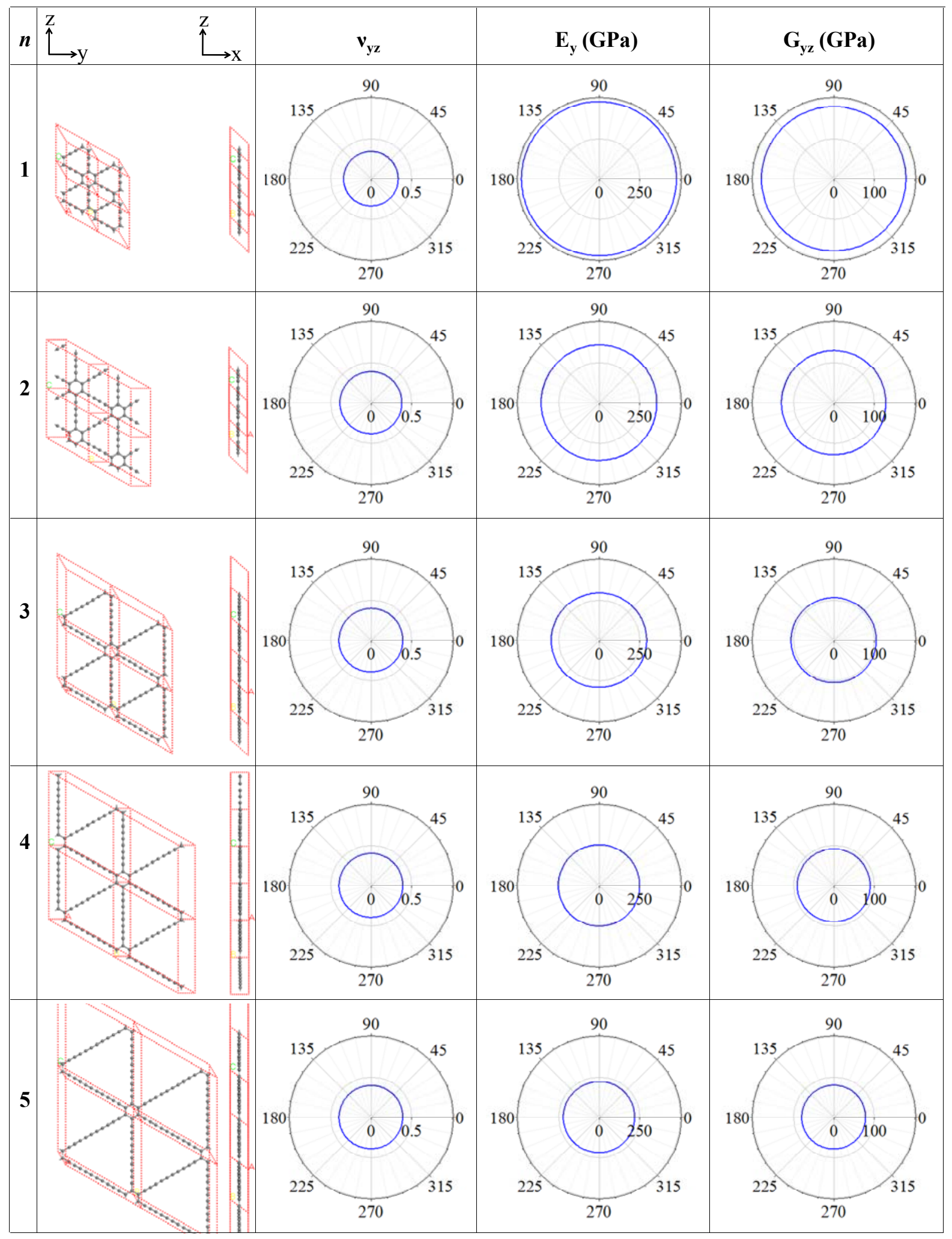

Figure S4: Structures and off-axis mechanical properties of the crystalline PA123456n systems as simulated by the PCFF force-field. 